\title{
Bending of floating flexible legs
}

\author{
KUN JOONG PARK AND HO-YOUNG KIM† \\ School of Mechanical and Aerospace Engineering, Seoul National University, Seoul 151-744, Korea
}

(Received 29 April 2008 and in revised form 19 June 2008)

When long thin flexible solid objects, such as the legs of water striders, disposable spoons and human hairs, are pressed against a liquid surface, they bend due to interfacial and hydrostatic forces. To understand the phenomenon, we study the bending of a sheet touching the liquid surface at an angle while clamped at the other end, to find its deflection and the load that the sheet can support before sinking. The theoretically predicted shapes of the sheet and the meniscus match well with experiments. Our theory shows that flexible sheets can support more load than rigid ones before sinking when the sheets are highly hydrophobic.

\section{Introduction}

Objects pushed against the free surface of liquid are either repelled from or drawn into the liquid due to interfacial tension. The interfacial tension acts along the contact line where the three phases of liquid/gas/solid meet, and the direction of the force is determined by the contact angle, which measures the relative affinity of the solid phase for the liquid to that for the gas. Tiny water-walking arthropods such as water striders having superhydrophobic legs use the interfacial tension or capillary force to stand on only tiptoes, to skate (Bush \& $\mathrm{Hu} 2006$ ) and to jump on the water surface (Lee \& Kim 2008). When the objects are long and flexible as observed in water strider legs and hairs covering those legs (Bush, Hu \& Prakash 2008), they deform under the capillary and hydrostatic forces. One can find a more mundane example of a similar kind when dining: a flexible spoon pushed onto soup surface is bent, requiring greater downward force before piercing the free surface than a rigid one. One also finds that it is extremely difficult to submerge individual hair in water due to its severe bending upon contact with the liquid.

The deformation of elastic filaments due to interfacial tension, termed elastocapillarity, has been studied for several different situations. Cohen \& Mahadevan (2003) showed that various equilibrium morphologies such as kinks, rings and rackets of carbon nanotubes and biological filaments arise due to competition between elastic and interfacial effects. Bico et al. (2004) and Kim \& Mahadevan (2006) investigated the coalescence of vertical flexible lamellae induced by capillary rise. Flexible horizontal beams have been shown to be deflected by a vapour bubble (Lee et al. 2007) and a liquid drop (Kwon et al. 2008) confined between the beam and a substrate because of the pressure jump across the gas/liquid interface caused by the surface tension effect. Neukirch et al. (2007) considered the piercing and buckling of initially immersed vertical elastic strips at the interface, where the perfectly wetting strip is compressed by surface tension. Song \& Sitti (2007) constructed an approximate numerical model 


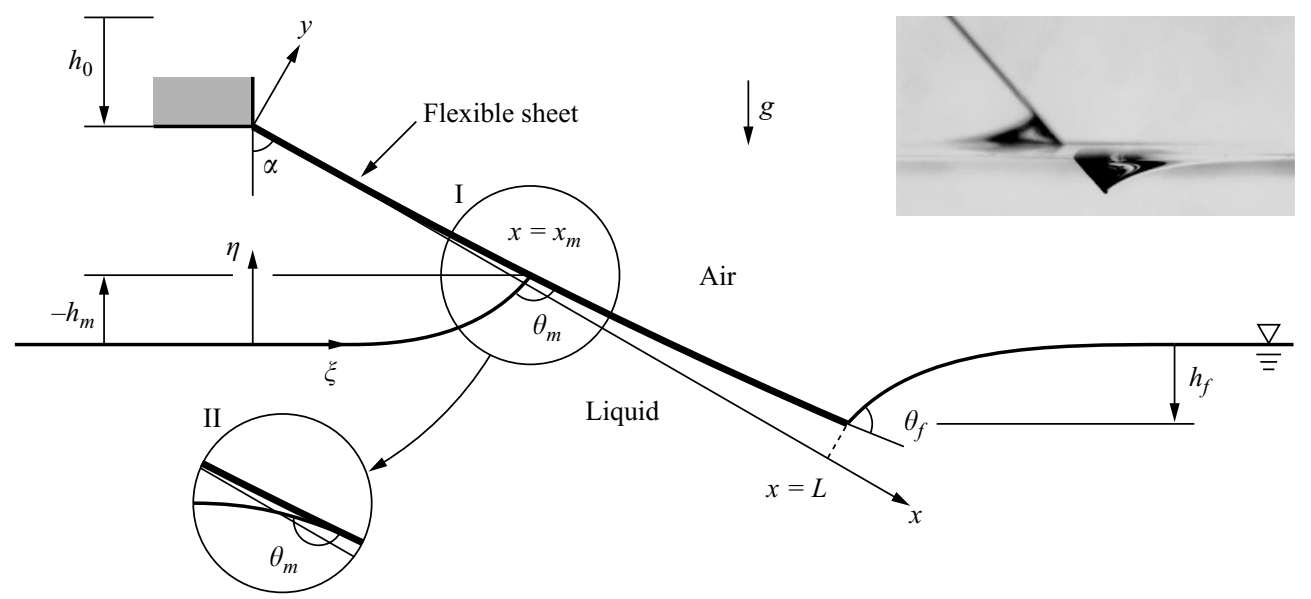

FIGURE 1. Schematic of a flexible sheet pushed against a liquid surface at an angle $\alpha$ to the vertical. The $x$-axis coincides with the undeformed sheet. The $\xi$ and $\eta$ coordinates denote the horizontal and the vertical, respectively. When the contact angle is not too large, the meniscus touching the down face is elevated (I), while the meniscus touching highly hydrophobic surfaces is depressed (II).

to estimate the bending and supporting load of a cylindrical flexible leg of a robot mimicking a water strider.

In seeking a physical understanding of the deformation of floating flexible filaments of varying wettability before penetrating liquid, here we consider the bending of a flexible sheet which pushes the free surface at an angle. We determine the deflection of the sheet by considering the interfacial tension and hydrostatic forces acting on the object. We calculate the maximum load that can be supported before the sheet pierces the liquid. We also experimentally measure sheet shapes and interface profiles to show that they agree quantitatively with our theory.

\section{Theoretical formulation, solution and experimental corroboration}

We consider a flexible sheet of length $L$, which pushes a liquid surface at an angle $\alpha$ to the vertical as illustrated in figure 1 . Since we are mainly interested in sheets lifted by the liquid surface, the solid surfaces are assumed to be hydrophobic. The sheet is clamped at one end $x=0$ while the other end at $x=L$ is free. Starting from a position where the sheet's free end just touches the liquid surface, the clamped end vertically descends a distance $h_{0}$, leading to the descent of the free end by $h_{f}$. Until penetration occurs, the up face of the sheet remains dry while the down face is wetted in the region $x_{m} \leqslant x \leqslant L$. The equilibrium conditions require force and torque be exerted at the clamped end to balance the surface tension force acting along the contact lines at $x=x_{m}$ and $x=L$ and the hydrostatic pressure force upon the wet face. We first consider the force and moment equilibrium for a rigid sheet and then formulate and solve the problem for a flexible sheet.

\subsection{Rigid sheet}

As a rigid sheet does not bend during its descent, $h_{0}=h_{f}$. The location of the liquid meniscus $x_{m}$ or the depression $h_{m}$, given by $h_{m}=h_{0}-\left(L-x_{m}\right) \cos \alpha$, can be obtained 
by integrating the Young-Laplace equation

$$
\rho g \eta=\sigma \frac{\eta^{\prime \prime}}{\left(1+\eta^{\prime 2}\right)^{3 / 2}},
$$

where $\rho$ is the liquid density, $g$ the gravitational acceleration, $\eta$ the elevation of the liquid surface from the unperturbed free surface, $\sigma$ the surface tension, and $\eta^{\prime} \equiv \mathrm{d} \eta / \mathrm{d} \xi$. Using the condition that $\eta=-h_{m}$ and $\eta^{\prime}=\cot \left(\theta_{m}-\alpha\right)$ at $x=x_{m}$, where $\theta_{m}$ is the contact angle at $x=x_{m}$, we obtain

$$
\left|h_{m}\right|=\sqrt{2} l_{c}\left[1-\sin \left(\theta_{m}-\alpha\right)\right]^{1 / 2},
$$

where the capillary length $l_{c}=(\sigma / \rho g)^{1 / 2}$. It can be shown that $h_{m}<0$, i.e. the meniscus is elevated from the unperturbed free surface, when $\left(\theta_{m}-\alpha\right)<\pi / 2$, and that $h_{m}>0$, i.e. the meniscus is depressed, when $\left(\theta_{m}-\alpha\right)>\pi / 2$. Note that as the sheet is lowered in a quasi-static manner, the meniscus touching the down face advances away from the sheet's free end, increasing the wet area. Therefore, $\theta_{m}$ always assumes the value of the critical advancing contact angle $\theta_{A, c}$, which was verified in our experiments described below. The depression of the meniscus at the free end, $h_{f}$, is given by

$$
h_{f}=\sqrt{2} l_{c}\left[1-\sin \left(\theta_{f}+\alpha\right)\right]^{1 / 2},
$$

because $\eta^{\prime}=-\cot \left(\theta_{f}+\alpha\right)$ at $x=L$. Since $h_{0}=h_{f}$ for a rigid sheet, (2.3) relates the descent distance $h_{0}$ to $\theta_{f}$. The contact angle at the free end $\theta_{f}$ increases as the sheet lowers, and the up surface of the sheet starts to be wet when $\theta_{f}$ reaches $\theta_{A, c}$ (Gibbs 1906; Cho et al. 2007), which condition gives the maximum distance of descent and the maximum load that the liquid surface can support before penetration.

Now we are ready to calculate the force and the moment applied at the clamped end by virtue of the equilibrium conditions. The force equilibrium conditions in the $x$ - and $y$-directions respectively give

$$
F_{v} \cos \alpha+F_{h} \sin \alpha+\sigma \cos \theta_{m}+\sigma \cos \theta_{f}=0
$$

and

$$
F_{v} \sin \alpha-F_{h} \cos \alpha+\sigma \sin \theta_{m}-\sigma \sin \theta_{f}+\int_{x_{m}}^{L} \rho g \eta(x) \mathrm{d} x=0,
$$

where $F_{v}$ and $F_{h}$ denote the force per unit width in the vertical and horizontal directions, respectively, and $\eta(x)$ is the vertical position of the sheet given by $\eta=(L-x)$ $\cos \alpha-h_{0}$. The last term on the left-hand side of (2.5) corresponds to the hydrostatic pressure force acting on the wet down face. The moment equilibrium gives the moment per unit width applied at the clamped end, $M_{0}$ :

$$
M_{0}=\sigma L \sin \theta_{f}-\sigma x_{m} \sin \theta_{m}-\int_{x_{m}}^{L} \rho g \eta(x) x \mathrm{~d} x .
$$

Eliminating $F_{h}$ in (2.4) and (2.5), we obtain the the scaled force $F_{v}$ :

$$
\frac{F_{v}}{\sigma}=-\frac{\cos \theta_{m}+\cos \theta_{f}}{\cos \alpha} .
$$

Although $F_{v} / \sigma$ appears to depend on the contact angles and $\alpha$ only, $\theta_{f}$ depends on $h_{0}$ and thus the descent distance of the sheet determines the force. The maximum value of the vertical force at the clamped end that the liquid surface can withstand before penetration is obtained by substituting $\theta_{A, c}$ for $\theta_{f}: F_{v, \max } / \sigma=-2 \cos \theta_{A, c} / \cos \alpha$. The maximum distance that the sheet can be lowered before penetration is given by (2.3) 
when $\theta_{f}=\theta_{A, c}$. We discuss the results for rigid sheets together with those for flexible sheets as described in the following.

\subsection{Flexible sheet}

Deflection of a flexible sheet alters the location of the meniscus touching the down face and the angles between the menisci and the sheet, leading to changes in the direction of the surface tension force and the hydrostatic force. We first estimate the perturbative change in the sheet shape and the force supported by the interface using dimensional and scaling analyses. The scaled characteristic sheet deflection $\delta / L$ must be a function of some dimensionless parameters. In addition to the scaled descent distance $h_{0} / L$, there are two other dimensionless length scales in the problem: the scaled capillary length $l_{c} / L$ and the scaled elastocapillary length $l_{e c} / L$, where $l_{e c}=(B / \sigma)^{1 / 2}, B$ being the flexural rigidity per unit width of the sheet. We also expect a dependence on the sheet inclination $\alpha$. Thus we may write $\delta / L=f\left(h_{0} / L, l_{c} / L, l_{e c} / L, \alpha, \theta\right)$, where we have added the contact angle $\theta$ to consider the orientation of the surface tension force. Balancing the torque exerted on a flexible sheet, $B \delta / L^{2}$, with the hydrostatic torque $\left(\sim \rho g h_{0}^{2} L \sec \alpha\right)$ yields $\delta / L \sim\left(h_{0} / l_{c}\right)^{2} \sec \alpha /\left(l_{e c} / L\right)^{2}$. When $l_{e c} / L \rightarrow \infty$, corresponding to the rigid sheet, $\delta / L \rightarrow 0$. Using $\delta \sin \alpha \sim h_{0}-h_{f}$, the descent distance of the free end is scaled as $h_{f} / l_{c} \sim h_{0} / l_{c}-k \lambda$, where $k$ is a constant and $\lambda=\left(h_{0} / L\right)^{2}\left(L / l_{c}\right)^{3}\left(L / l_{e c}\right)^{2} \tan \alpha$. The vertical forces due to the hydrostatic pressure on the flexible sheet and on the rigid sheet are scaled as $F_{v, f} \sim \rho g h_{f}^{2} \tan \alpha$ and $F_{v, r} \sim \rho g h_{0}^{2} \tan \alpha$, respectively. Thus the ratio of the vertical force on a flexible sheet to that on a rigid sheet becomes $F_{v, f} / F_{v, r} \sim\left[1-c\left(h_{0} / L\right)\left(L / l_{c}\right)^{2}\left(L / l_{e c}\right)^{2} \tan \alpha\right]^{2}$, where $c$ is a constant. To go beyond these scaling estimates and fully consider the forces acting on the sheet, we formulate and solve a free boundary problem as follows.

When $\delta / L \ll 1$, we may use a geometrically and physically linear theory of a sheet to describe its transverse deformation $y(x)$ (Landau \& Lifshitz 1986):

$$
B y^{\mathrm{iv}}=\mathscr{H}\left(x-x_{m}\right)\left(-\rho g \eta+q_{s}\right),
$$

where $y^{\prime} \equiv \mathrm{d} y / \mathrm{d} x, \mathscr{H}($.$) is the Heaviside function, and \eta(x)$ is the vertical position of the sheet, given by $\eta=(L-x) \cos \alpha-h_{0}+y \sin \alpha$. Here we include the capillary force $q_{s}$ at the two side edges of the sheet to give a correction to the idealized two-dimensional model assuming an infinite sheet width. The Young-Laplace equation for the menisci touching the side edges in the plane perpendicular to the $x$-axis gives

$$
q_{s}(x)=-\frac{2 \rho g \eta l_{c} \sin \alpha}{w}\left(1-\frac{\eta^{2}}{4 l_{c}^{2}}\right)^{1 / 2},
$$

where $w$ is the width of the sheet. The magnitude of $q_{s}$ can be scaled as $\rho g \eta l_{c} \sin \alpha / w$, and thus is negligible compared to $\rho g \eta$ for $w \gg l_{c}$, which will be assumed to be valid henceforth. To complete the formulation of the problem, we need to specify some boundary and matching conditions and determine the eight constants of integration, the unknown meniscus location $x_{m}$ and the contact angle at the free end $\theta_{f}$. The sheet is clamped at $x=0$ so that the corresponding boundary conditions are $y(0)=0$ and $y^{\prime}(0)=0$. At the other end, $x=L$, the sheet is free of torque, but is subject to a transverse shear force due to surface tension. Therefore, we may write: $y^{\prime \prime}(L)=0$ and $B y^{\prime \prime \prime}(L)=-\sigma \sin \left(\theta_{f}+\phi_{f}\right)$, where $\phi_{f}=\tan ^{-1}\left(\left.y^{\prime}\right|_{x=L}\right)$. These four boundary conditions must be supplemented by matching conditions at the meniscus $x=x_{m}$ given by the continuity of the deflection, the slope, and the curvature of the sheet, i.e. $[y]=\left[y^{\prime}\right]=\left[y^{\prime \prime}\right]=0$ where $[A]=\lim _{\epsilon \rightarrow 0}\left\{A\left(x_{m}+\epsilon\right)-A\left(x_{m}-\epsilon\right)\right\}$. However, there is a jump in the transverse shear force across $x=x_{m}$ due to surface tension so that 
$\left[B y^{\prime \prime \prime}\right]=-\sigma \sin \left(\theta_{m}-\phi_{m}\right)$, where $\phi_{m}=\tan ^{-1}\left(\left.y^{\prime}\right|_{x=x_{m}}\right)$. The additional two conditions are provided by describing the interface profiles at $x=x_{m}$ and $x=L$ using the YoungLaplace equation, respectively:

$$
\begin{gathered}
\left|h_{m}\right|=\left|h_{0}-\left(L-x_{m}\right) \cos \alpha-y\left(x_{m}\right) \sin \alpha\right|=\sqrt{2} l_{c}\left[1-\sin \left(\theta_{m}-\phi_{m}-\alpha\right)\right]^{1 / 2}, \\
h_{f}=h_{0}-y(L) \sin \alpha=\sqrt{2} l_{c}\left[1-\sin \left(\theta_{f}+\phi_{f}+\alpha\right)\right]^{1 / 2} .
\end{gathered}
$$

We note that the effects of the sheet bending on the change of interface profile compared with those corresponding to the rigid sheets are manifested through the deflection angles $\phi_{m}$ and $\phi_{f}$ appearing in (2.10) and (2.11), respectively.

To make the equations and boundary conditions dimensionless, we use the scaled variables $x=L \hat{x}, y=L \hat{y}, \eta=L \hat{\eta}, x_{m}=L \hat{x}_{m}, h_{0}=L \hat{h}_{0}, h_{m}=L \hat{h}_{m}$, and $h_{f}=L \hat{h}_{f}$, so that the complete boundary value problem is

$$
\hat{y}^{\mathrm{iv}}=-\frac{L^{4} \hat{\eta}}{l_{e c}^{2} l_{c}^{2}} \mathscr{H}\left(\hat{x}-\hat{x}_{m}\right),
$$

subject to the conditions

$$
\left.\begin{array}{l}
\hat{y}(0)=0, \quad \hat{y}^{\prime}(0)=0, \\
\hat{y}^{\prime \prime}(1)=0, \quad \hat{y}^{\prime \prime \prime}(1)=-L^{2} \sin \left(\theta_{f}+\phi_{f}\right) / l_{e c}^{2}, \\
{[\hat{y}]=0, \quad\left[\hat{y}^{\prime}\right]=0,} \\
{\left[\hat{y}^{\prime \prime}\right]=0, \quad\left[\hat{y}^{\prime \prime \prime}\right]=-L^{2} \sin \left(\theta_{m}-\phi_{m}\right) / l_{e c}^{2},} \\
\left|\hat{h}_{0}-\left(1-\hat{x}_{m}\right) \cos \alpha-\hat{y}\left(\hat{x}_{m}\right) \sin \alpha\right|=\sqrt{2} l_{c}\left[1-\sin \left(\theta_{m}-\phi_{m}-\alpha\right)\right]^{1 / 2} / L, \\
\hat{h}_{0}-\hat{y}(1) \sin \alpha=\sqrt{2} l_{c}\left[1-\sin \left(\theta_{f}+\phi_{f}+\alpha\right)\right]^{1 / 2} / L .
\end{array}\right\}
$$

We see that the shape of the sheet $\hat{y}(\hat{x})$ depends on five dimensionless parameters, namely $h_{0} / L, l_{c} / L, l_{e c} / L, \alpha$ and the contact angle $\theta_{m}=\theta_{A, c}$, consistent with the dimensional analysis result above. Integrating (2.12) yields a polynomial shape of the sheet with eight integration constants. These constants, the unknown meniscus location $\hat{x}_{m}$ and the contact angle $\theta_{f}$ are obtained by using ten conditions (2.13) to yield closed-form expressions for $\hat{y}(\hat{x})$, employing MATLAB.

Figure 2 shows the shapes of the sheets of different wettability $\theta_{A, c}$ upon solving the foregoing equations for different descent distances $h_{0} / L$. Lowering the sheet increases the wetted area with the meniscus at the down face moving toward the clamped end, and causes the sheet to bend upward owing to the increased hydrostatic force and the vertical component of the surface tension at the free end. It is interesting to note that for $\theta_{A, c}$ not significantly exceeding a right angle, the sheet can be bent downward when it initially contacts the liquid surface, corresponding to relatively small $h_{0} / L$ as shown in figure $2(b)$. It is because the downward surface tension force at $x=x_{m}$ and the downward hydrostatic force on the wet region above the unperturbed free surface dominate the other forces in this case. In figure 3 , we show the dimensionless shapes of the sheet as a function of the following two dimensionless parameters: $l_{e c} / L$ and $\theta_{A, c}$. The trends are intuitively obvious. The sheet bends more as the sheet is relatively more flexible (decreased $l_{e c} / L$ ) and more hydrophobic (increased $\theta_{A, c}$ ). Unlike the foregoing parameters, the dependence of the sheet deflection on the remaining two parameters, $l_{c} / L$ and $\alpha$, is rather complicated as shown in figure 4 . While the sum of the torques by the hydrostatic pressure and the surface tension forces monotonically 

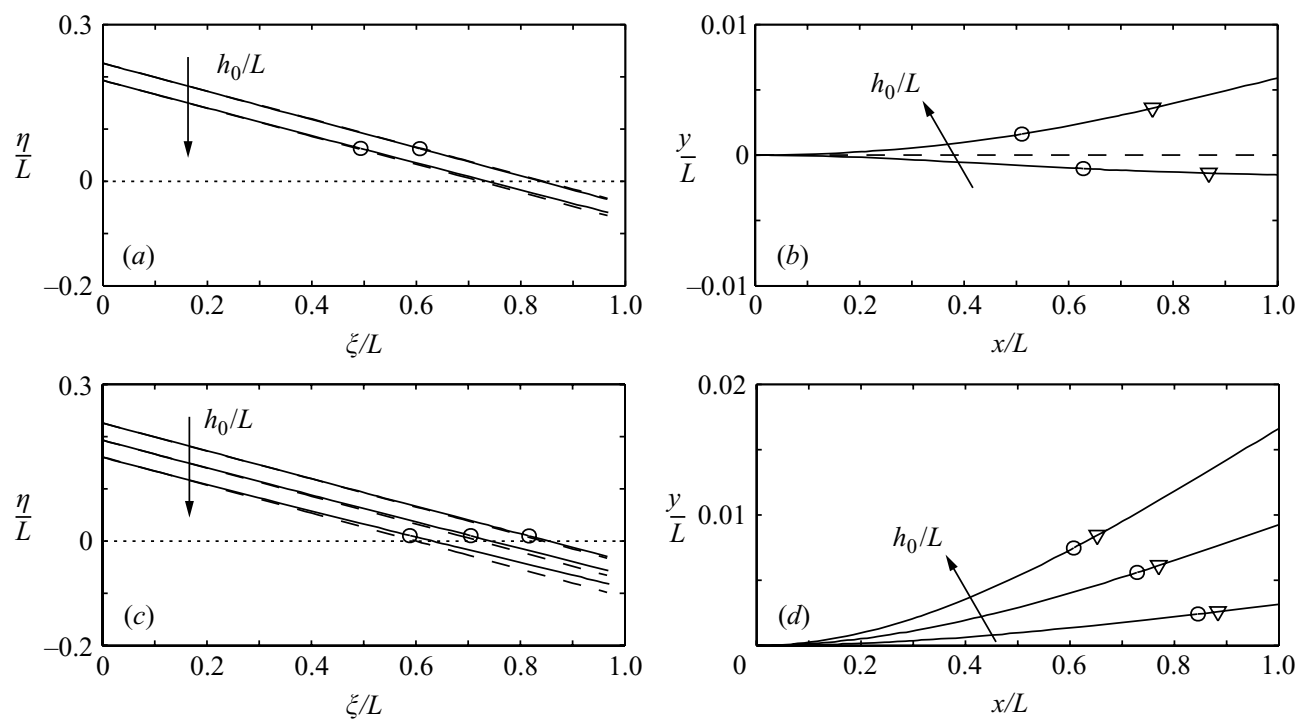

FIGURE 2. Equilibrium shapes of floating flexible sheets (solid lines) versus $h_{0} / L$. The dotted and dashed lines denote the unperturbed free surface and the rigid sheets, respectively. Circles and triangles respectively denote the locations of the meniscus at $x=x_{m}$ and the locations where the sheets intersect with the unperturbed free surface, $\eta=0$. The values of the parameters used in the computations are such that $l_{c} / L=0.0545, l_{e c} / L=9.68$ and $\alpha=75^{\circ}$, corresponding to the parameter values for a sheet of length $L=50 \mathrm{~mm}$ and $B=0.017 \mathrm{~N} \mathrm{~m}$ floating on water with $\sigma=0.0728 \mathrm{~N} \mathrm{~m}^{-1}$. (a) The shape of the sheet with $\theta_{A, c}=95^{\circ}$. In the direction of the arrow, the parameter $h_{0} / L$ increases taking the values 0.0327 and 0.0655 . (b) The sheet shapes of (a) replotted in $(x, y)$-coordinates. (c) The shape of the sheet with $\theta_{A, c}=155^{\circ}$. The parameter $h_{0} / L$ takes the values $0.0327,0.0655$, and 0.0982 , in the direction of the arrow. $(d)$ The sheet shapes of $(c)$ replotted in $(x, y)$-coordinates.
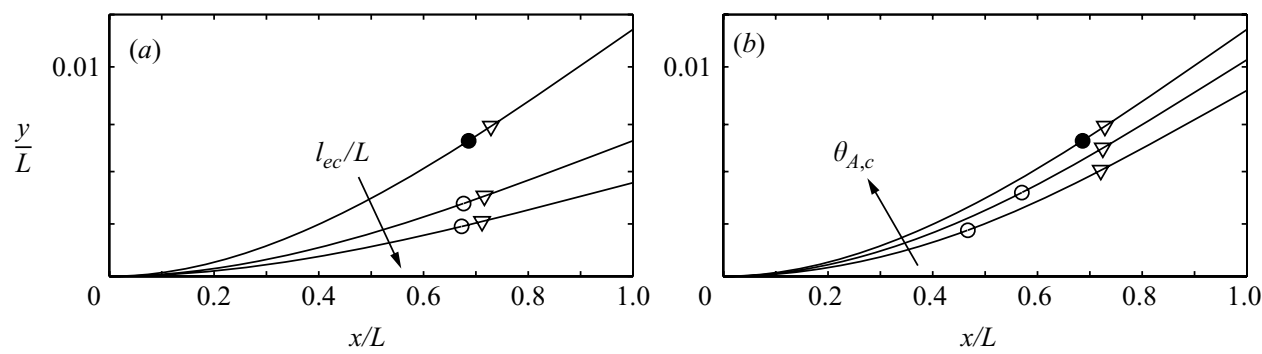

FIGURE 3. Effects of $(a) l_{e c} / L$ and $(b) \theta_{A, c}$ on the sheet shape (solid lines) and the location of the meniscus at $x=x_{m}$ (circles). Triangles denote the locations where the sheets intersect with the unperturbed free surface. For comparison, the curve with a filled circle in each case corresponds to the parameter values $h_{0} / L=0.0771, l_{c} / L=0.0545, l_{e c} / L=9.68, \alpha=75^{\circ}$ and $\theta_{A, c}=155^{\circ}$. In the direction of the arrow, $(a) l_{e c} / L$ increases taking the values $9.68,13.7$, and 16.8 ; and $(b) \theta_{A, c}$ increases taking the values $95^{\circ}, 125^{\circ}$, and $155^{\circ}$.

increases with the increase of $h_{0} / L, L / l_{e c}$ and $\theta_{A, c}$ for the entire parameter space, the resultant torque may increase (figures $4 b$ and $4 c$ ) or decrease (figures $4 a$ and $4 d$ ) with increasing $l_{c} / L$ or $\alpha$ depending on the other parameter values.

In addition to the sheet shapes and the locations of the menisci, the force that the liquid surface supports at a given depression distance $h_{0}$ can be obtained using our theory. Before treating this force, we turn to a comparison of the theory for 

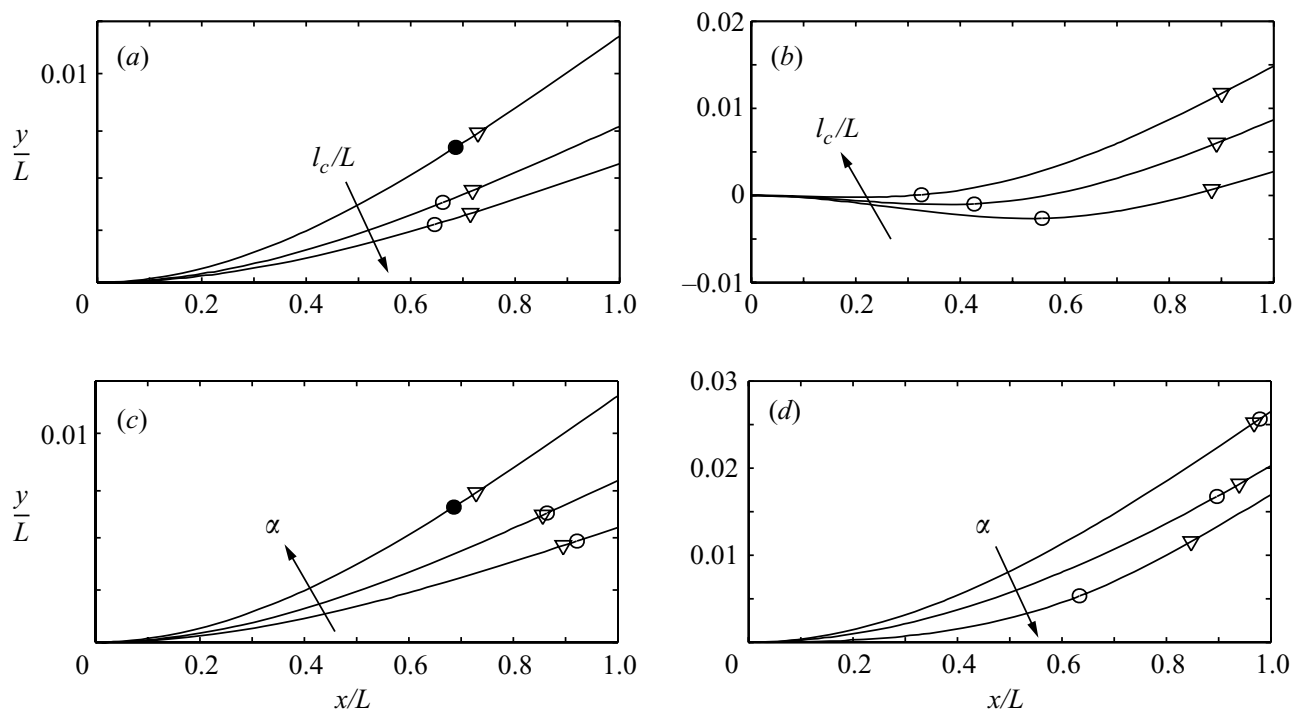

Figure 4. Effects of $(a, b) l_{c} / L$ and $(c, d) \alpha$ on the shape of the sheet (solid lines) and the location of the contact line at $x=x_{m}$ (circles). Triangles denote the locations where the sheets intersect with the unperturbed free surface. To show the effects of changing $l_{c} / L$, in the direction of the arrow, $(a) l_{c} / L$ increases taking the values $0.0545,0.0771$, and 0.0945 , with $h_{0} / L=0.0771, l_{e c} / L=9.68, \alpha=75^{\circ}$ and $\theta_{A, c}=155^{\circ}$; and $(b) l_{c} / L$ increases taking the values $0.173,0.244$, and 0.299 , with $h_{0} / L=0.0599, l_{e c} / L=2.16, \alpha=60^{\circ}$ and $\theta_{A, c}=95^{\circ}$. To show the effects of changing $\alpha$, in the direction of the arrow, $(c) \alpha$ increases taking the values $45^{\circ}, 60^{\circ}$, and $75^{\circ}$, with $h_{0} / L=0.0771, l_{c} / L=0.0545, l_{e c} / L=9.68$ and $\theta_{A, c}=155^{\circ}$; and $(d) \alpha$ increases taking the values $60^{\circ}, 70^{\circ}$, and $80^{\circ}$, with $h_{0} / L=0.0379, l_{c} / L=0.109, l_{e c} / L=1.53$ and $\theta_{A, c}=155^{\circ}$.

sheet bending and meniscus deformation with experiments. The flexible sheets were rectangular glass sheets $24 \mathrm{~mm}$ wide and $0.14 \mathrm{~mm}$ thick. The sheet was clamped at various angles $\alpha$ while the effective sheet length was kept at $L=50 \mathrm{~mm}$. The sheet surfaces were coated with two materials to vary $\theta_{A, c}$ with deionized water, the liquid used in the experiments. We obtained $\theta_{A, c}=95^{\circ}$ by spray-coating the sheets with a nitrocellulose lacquer, and $\theta_{A, c}=155^{\circ}$ with a mixture of chloroform and melted alkyl ketene dimer (AKD). At a fixed $\alpha$, the sheet was lowered slowly by a linear stage until the sheet penetrates the water surface. The images of the sheet and the liquid menisci were taken by a CCD (charge coupled device) camera with resolution of $512 \times 512$ pixels. Figure 5 shows representative images before, upon and after penetration of the sheet through water surface.

While lowering the sheet, we measured the depression $h_{f}$ and the contact angle $\theta_{f}$ at the free end, and the values are smaller than those for rigid sheets at the same $h_{0}$ due to bending. We see excellent agreement between the theory and the experiments in figure 6. In particular, figure $6(b)$ shows that the penetration occurs upon $\theta_{f}$ reaching $\theta_{A, c}$ and that the meniscus on the up face of the sheet slowly advances with a contact angle fairly close to $\theta_{A, c}$ as the sheet is further lowered. In the inset of figure $6(a)$, we compare the experiments with the scaling introduced above: $h_{f} / l_{c} \sim h_{0} / l_{c}-k \lambda$, and find good agreement owing to the dominant hydrostatic pressure effect on the deflection in this case. Figure 7 shows the depression of the clamped end $h_{0, p} / l_{c}$ and the free end $h_{f, p} / l_{c}$ at the moment of penetration. Besides good agreement between the experiments and the theory, we see that bending of the sheet allows the clamped end to descend further than a rigid sheet, and the increase of $h_{0, p} / l_{c}$ is more pronounced 
(a)

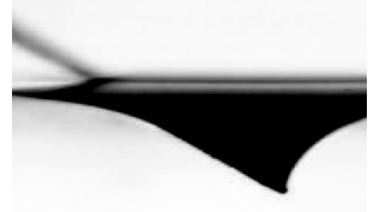

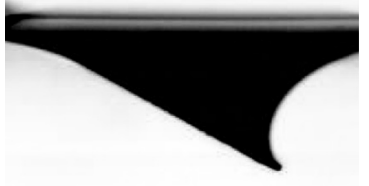

(b)

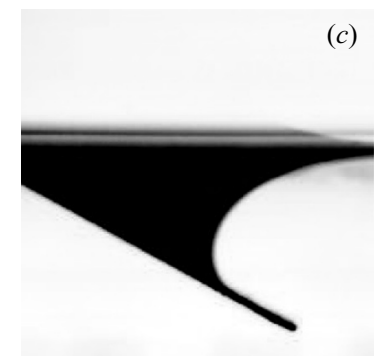

$3 \mathrm{~mm}$

FIGURE 5. Images of a flexible sheet, coated with a mixture of chloroform and melted AKD and clamped at $\alpha=60^{\circ}$ to the left, pushing the water surface down. As the sheet is lowered from $(a)$ to $(b)$, the contact angle at the free end $\theta_{f}$ increases until it finally reaches $\theta_{A, c}$ when the up face of the sheet begins to be wetted (onset of penetration). (a) Before piercing: $h_{0}=3.8 \mathrm{~mm}, h_{f}=3.4 \mathrm{~mm}$ and $\theta_{f}=110^{\circ}$. (b) Upon piercing: $h_{0}=5.3 \mathrm{~mm}, h_{f}=4.8 \mathrm{~mm}$ and $\theta_{f}=155^{\circ} .(c)$ After piercing: $h_{0}=6.8 \mathrm{~mm}, h_{f}=6.3 \mathrm{~mm}$ and $\theta_{f}=156^{\circ}$.
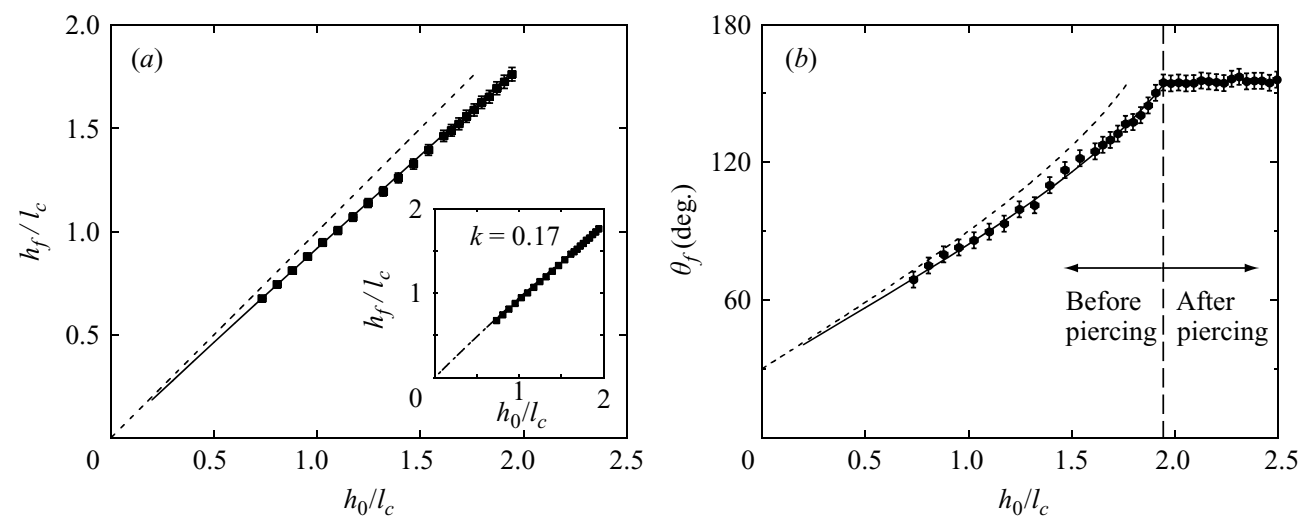

FIGURE 6. Comparison of the experimental measurements (filled symbols) and theoretical predictions (solid lines) of $(a) h_{f} / l_{c}$ and $(b) \theta_{f}$ versus $h_{0} / l_{c}$ for a flexible sheet with $l_{c} / L=0.0545, l_{e c} / L=9.68, \alpha=60^{\circ}$ and $\theta_{A, c}=155^{\circ}$. Dotted lines correspond to the predicted results for a rigid sheet. Inset: the experimental results and the scaling law $h_{f} / l_{c} \sim h_{0} / l_{c}-k \lambda$.

with the increase of $\alpha$. However, the location of the free end $h_{f, p} / l_{c}$ at penetration varies little because the slope perturbation $\phi_{f}$ in (2.11) is much smaller than $\theta_{A, c}+\alpha$.

The force balance equations similar to (2.4) and (2.5) give the vertical downward force $F_{v}$ at the clamped end $x=0$. The computed results for the dependence of the vertical force on $h_{0} / l_{c}$ and $\alpha$ are shown for the sheets with different $\theta_{A, c}$ in figure 8 . As was observed in figures $2(a)$ and $2(b)$, even when the sheet is hydrophobic with $\theta_{A, c}>90^{\circ}$, the tilted sheet tends to be drawn into the liquid for small $h_{0} / l_{c}$ unless $\theta_{A, c}$ is very large. Thus the force values in figure $8(a)$, corresponding to $\theta_{A, c}=95^{\circ}$, take negative values for small $h_{0} / l_{c}$, implying that moderately hydrophobic sheets need to be externally supported (at the clamped end) upward to prevent them from sinking when they push on the liquid surface. As was explained earlier, the meniscus touching the down face at $x=x_{m}$ results in downward surface tension and hydrostatic pressure forces, which dominate the upward forces elsewhere. Alternatively, one can consider the forces in the context of Keller's (1998) theorem stating that the net vertical force exerted on a floating body is equal to the weight of the liquid volume displaced by 

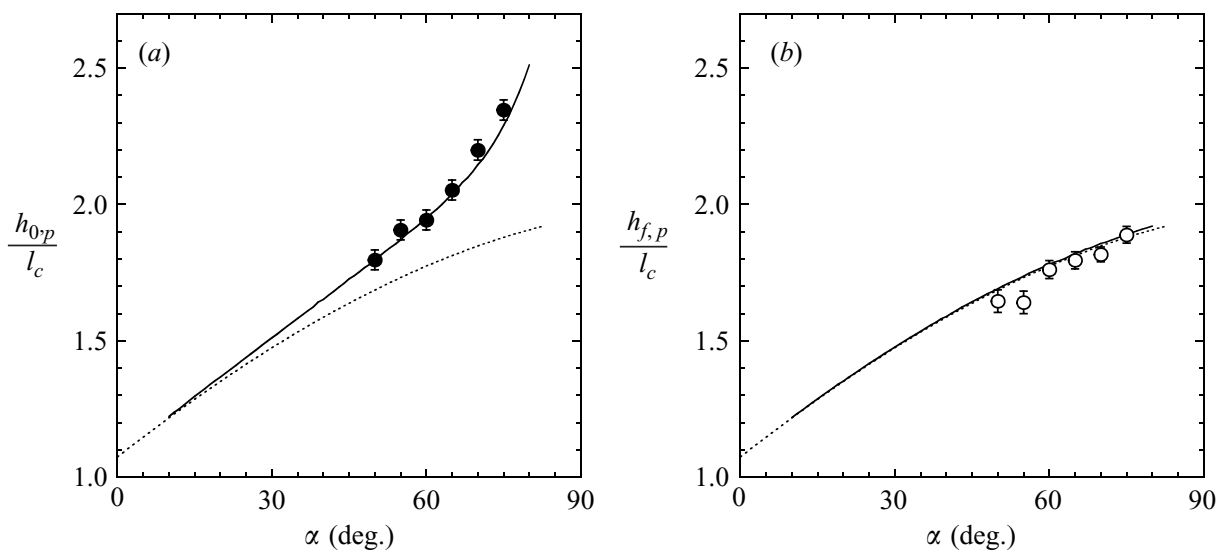

FiguRE 7. Depression distances of $(a)$ the clamped end and $(b)$ the free end upon piercing $\left(\theta_{f}=\theta_{A, c}\right)$, obtained experimentally (circles) and theoretically (solid lines) versus $\alpha$. Dotted lines denote the predictions for a rigid sheet. The parameter values are $l_{c} / L=0.0545, l_{e c} / L=9.68$ and $\theta_{A, c}=155^{\circ}$. Each curve ends at a high $\alpha$, where the clamped end is wet due to the elevated meniscus at $x=x_{m}$.
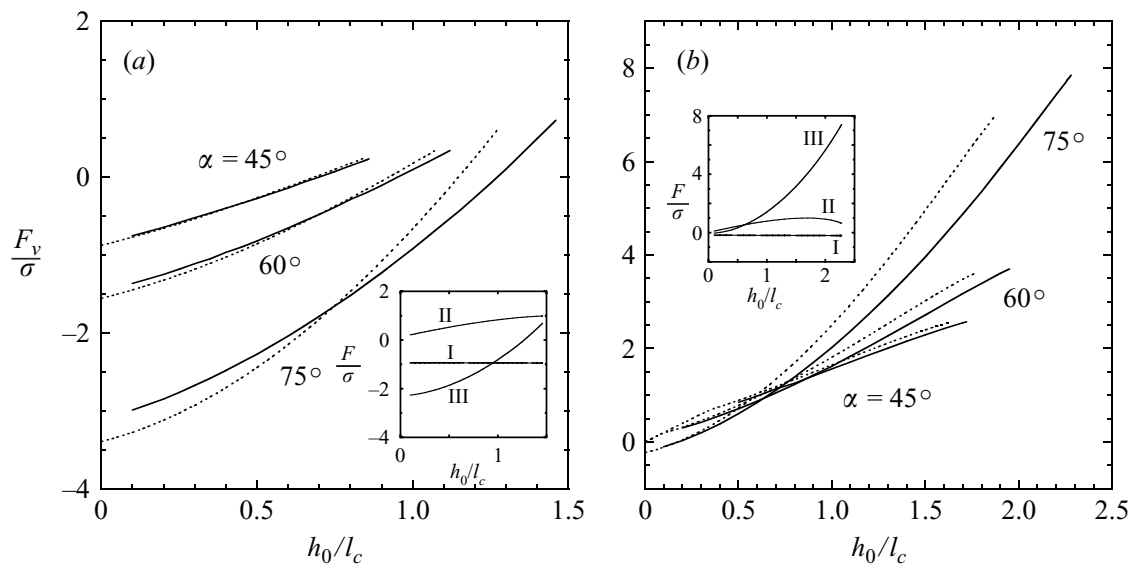

FiguRE 8. Downward vertical force per unit width exerted at the clamped end in static equilibrium (solid lines) with the parameter values $l_{c} / L=0.0545$ and $l_{e c} / L=9.68$. Dotted lines correspond to the vertical force acting on a rigid sheet with the same $l_{c} / L$. Each curve ends when $\theta_{f}$ reaches $\theta_{A, c}$, onset of penetration. (a) $\theta_{A, c}=95^{\circ}$. (b) $\theta_{A, c}=155^{\circ}$. Insets: contribution of each force component to the total force. (I) The surface tension force at the contact line on the down face; (II) the surface tension force at the free end; and (III) the hydrostatic pressure force, for $\alpha=75^{\circ}$.

the meniscus and by the solid body. The downward force due to the lifting of the meniscus at $x=x_{m}$ is not balanced until $h_{0} / l_{c}$ becomes sufficiently large that the weight of the displaced liquid volume facing the up face equals that of the elevated water weight behind the down face. However, when the sheet is superhydrophobic as in figure $8(b)$, it is supported upward by a liquid surface in most configurations and the magnitude of the force increases with $h_{0} / l_{c}$. Comparing the maximum load that the liquid surface can support just before the penetration occurs, little difference between the flexible and rigid sheets is observed in figure $8(a)$, when the sheets are moderately hydrophobic. However, as seen in figure $8(b)$, one needs to exert a greater force to sink flexible sheets than rigid ones when the sheets are highly hydrophobic. 
Although the rigid sheets support more load than the flexible ones under the same depression distance $h_{0} / l_{c}$, the flexible sheets can be depressed deeper before $\theta_{f}$ reaches $\theta_{A, c}$, leading to an increase of the displaced volume of liquid. The inset of figure $8(b)$ reveals that the hydrostatic pressure force is dominant over surface tension forces when a superhydrophobic sheet is lowered a considerable amount.

\section{Discussion}

We have presented a theory to predict the deflection of floating flexible sheets clamped at one end and corroborated the results using experiments. Further, we calculated the force at the clamped end, to find that flexible sheets can support more load than rigid ones when the sheets are highly hydrophobic owing to increased volume of the displaced liquid. Our focus in this paper has been on the sheet-shaped objects. Thus when one considers the bending of straight or tapered cylinders, such as the legs of water-walking arthropods and hairs covering those legs, the theory needs to be modified. For long thin cylinders, the surface tension force acting along the cylinder side dominates the hydrostatic force. A scaling law can be readily constructed for the deformation $\delta$ of a cylinder of length $L$ and diameter $D$ lowered a distance $h_{0}$ from the unperturbed free surface with clamped angle $\alpha$, using the balance of the torque due to surface tension $\sigma h_{0} L \tan \alpha$ with $B D \delta / L^{2}$, where $B$ is the bending stiffness per unit diameter of the cylinder. We then obtain $\delta / L \sim\left(h_{0} / D\right)\left(L / l_{e c}\right)^{2} \tan \alpha$. The vertical forces due to the surface tension along the side of a flexible cylinder and of a rigid cylinder are scaled as $F_{v, f} \sim \rho g h_{f}^{2} l_{c} \tan \alpha$ and $F_{v, r} \sim \rho g h_{0}^{2} l_{c} \tan \alpha$, respectively. Thus $F_{v, f} / F_{v, r} \sim\left[1-b(L / D)\left(L / l_{e c}\right)^{2} \tan \alpha \sin \alpha\right]^{2}$, where $b$ is a constant. A rigorous free boundary problem needs to be solved to obtain the detailed solutions of the shapes of the cylinder and the surrounding menisci and the force, which requires complicated geometric considerations for the contact of the meniscus with the circular cylinder wall. The maximum load that the cylinder can support before sinking can be obtained when the critical sinking condition of the cylinder is determined. Our theory can also be extended to more generalized floating situations in addition to the current obliquely clamped end condition.

This work was supported by a Korea Research Foundation grant (KRF-2007-412J03001) administered via SNU-IAMD.

\section{REFERENCES}

Bico, J., Roman, B., Moulin, L. \& Boudaoud, A. 2004 Nature 432, 690.

Bush, J. W. M. \& Hu, D. L. 2006 Annu. Rev. Fluid Mech. 38, 339-369.

Bush, J. W. M., Hu, D. L. \& Prakash, M. 2008 Adv. Insect Physiol. 34, 117-192.

Cho, H., Kim, H.-Y., Kang, J. Y. \& Kim, T. S. 2007 J. Colloid Interface Sci. 306, 379-385.

Cohen, A. E. \& Mahadevan, L. 2003 Proc. Natl Acad. Sci. 100, 12141-12146.

GibBS, J. W. 1906 Scientific Papers, vol. 1, Dover, p. 326.

Keller, J. B. 1998 Phys. Fluids 10, 3009-3010.

Kim, H.-Y. \& MahadeVAN, L. 2006 J. Fluid Mech. 548, 141-150.

Kwon, H.-M., Kim, H.-Y., Puëll, J. \& Mahadevan, L. 2008 J. Appl. Phys. 103, 093519.

Landau, L. D. \& Lifshitz, E. M. 1986 Theory of Elasticity, 3rd Edn. Pergamon.

LeE, D.-G. \& Kim, H.-Y. 2008 Langmuir 24, 142-145.

Lee, H. J., Chang, Y. S., Lee, Y. P., Jeong, K.-H. \& Kim, H.-Y. 2007 Sens. Actuators A 136, 717-722.

Neukirch, S., Roman, B., De Gaudemaris, B. \& Bico, J. 2007 J. Mech. Phys. Solids 55, 1212-1235.

Song, Y. S. \& SitTi, M. 2007 IEEE Trans. Robot. 23, 578-589. 